\title{
ON THE PRIVATE PROVISION OF PUBLIC GOODS
}

\author{
Theodore BERGSTROM, Lawrence BLUME and Hal VARIAN* \\ Department of Economics, University of Michigan, Ann Arbor, MI 48109, USA
}

Received January 1984, revised version received August 1985

\begin{abstract}
We consider a general model of the non-cooperative provision of a public good. Under very weak assumptions there will always exist a unique Nash equilibrium in our model. A small redistribution of wealth among the contributing consumers will not change the equilibrium amount of the public good. However, larger redistributions of wealth will change the set of contributors and thereby change the equilibrium provision of the public good. We are able to characterize the properties and the comparative statics of the equilibrium in a quite complete way and to analyze the extent to which government provision of a public good 'crowds out' private contributions.
\end{abstract}

\section{Introduction}

A standard result of the theory of public goods is that, in general, pure public goods would bc undcrsupplied by voluntary contributions. Nevertheless, there appear to be many important instances in which pure public goods are voluntarily supplied. Aside from the obvious example of private donations to charity, ${ }^{1}$ the campaign funds of political parties and the political action funds of special interest groups are, to a large extent, financed by voluntary contributions. As Becker (1981) has pointed out, much of the economic activity of the family unit must be explained as the outcome of voluntary contributions. Kemp (1984a, 1984b) suggests applications of this theory to the issues of multilateral 'foreign aid' and of the provision of 'international public goods'.

The first substantial contribution to the theory of voluntary provision of public goods of which we are aware was due to Olson (1965). Recently, there have been several interesting theoretical discussions of this issue. These include papers by Chamberlin (1974, 1976), McGuire (1974), Abrams and

\footnotetext{
*This work was supported in part by the National Science Foundation. We wish to thank Joseph Greenberg, Peter Hartley, Jonathan Pincus, Russell Roberts, and an anonymous referee for helpful comments.

${ }^{1}$ According to Roberts (1984), private donations to charity in the United States in 1981 totalled about 53 billion dollars. This is about 2 percent of GNP or about 225 dollars per capita. Roberts suggests, however, that some measured charitable giving may actually be disguised private consumption.
} 
Schmitz (1978), Becker (1981), Laffont (1982), Young (1982), Warr (1982, 1983), Brennan and Pincus (1983), Cornes and Sandler (1984, 1985), Kemp (1984a, 1984b), and Roberts (1984). In addition, several researchers have conducted experiments to determine whether subjects are likely to 'free ride' in an environment where pure public goods are supplied voluntarily. Among those who have contributed experimental evidence are Smith (1980), Marwell and Ames (1981), Isaac, McCue and Plott (1982), and Kim and Walker (1984). Kim and Walker report on the results of several other related experimental tests and offer an interesting theoretical explanation of apparently contradictory findings among these studies.

Most of the theoretical and experimental work mentioned above has concerned models in which a single pure public good is supplied. Each consumer's preferences are assumed to depend on his private consumption and the sum of everyone's voluntary contributions. The theoretical papers have for the most part deal with 'Nash equilibrium' in which each consumer assumes that the contributions of others will be independent of his own. The experimental papers have been concerned with the question of whether behavior is consistent with the Nash hypothesis. Sugden (1982) argues that the predictions of the Nash equilibrium model with a pure public good seem inconsistent with empirical examples of charities to which there are many donors. ${ }^{2}$ Cornes and Sandler (1984), Steinberg (1984), and Andreoni (1985) treat a model in which a consumer's utility depends not only on the aggregate amount of contributions, but also on his own contribution. We agree with these authors that a complete descriptive model of charitable contributions should allow each consumer to be concerned about his own contribution as well as the aggregate supply of the public good. Even where the good supplied is a pure public good and where the contributions of different individuals are perfect substitutes from a "technical standpoint, a fully satisfactory model should probably accommodate preferences of people who feel a 'warm glow' from having 'done their bit'. Furthermore, in many instances of voluntarily supplied public goods, the contributions of individuals are public information. Those who desire the good opinion of their neighbors may believe the size of their own contributions to have an importance beyond their effect on total supply.

Despite our interest in a more general model we confine our attention in this paper to the case where people are concerned only about their private consumptions and the total supply of public goods. This is the model which has received the most attention so far in the literature and is, we suspect, the one on which many economists base their intuitions. The equilibrium theory for this polar case is strikingly clean and decisive, yet contains some

\footnotetext{
${ }^{2} \mathrm{He}$ suggests abandoning the hypothesis of utility maximization in favor of an ethically based rule of behavior.
} 
interesting surprises. A thorough understanding of this case is, we think, a natural step on the way to a general theory.

Warr (1983) discovered a striking result concerning the provision of a public good in a voluntary Nash equilibrium: ${ }^{3}$

When a single public good is provided at positive levels by private individuals, its provision is unaffected by a redistribution of income. This holds regardless of differences in individual preferences and despite differences in marginal propensities to contribute to the public good.

As Warr acknowledges, his proof depends on calculus first-order conditions, and therefore applies only to a redistribution of income among current contributors which does not alter the composition of the contributing set. As Warr also remarks, his result is seriously limited in its applications because it only deals with the case of a single public good. However, Kemp (1984a) suggests a way to extend Warr's results to the case of more than one public good. In section 2 of this paper we sharpen and generalize Warr's neutrality theorem. In section 6 we improve on Kemp's neutrality result for the case of multiple public goods.

Interesting though it is, the importance of the neutrality theorem should not be overstated. Income redistribution among contributors will not change the supply of a public good if it does not change the set of contributing consumers. But, as we will show, many of the interesting applications involve income redistribution that changes the set of contributing consumers and/or alters the wealth of the current set of contributing consumers.

The message that emerges from our study is that adjustments on the 'extensive margin' - the decision of whether or not to become a contributor are at least as important as adjustments on the 'intensive margin' - the decision of how much to contribute. In general, only a small subset of consumers will actually contribute to the public good, and changes in the wealth distribution will only have a significant effect on the provision of the public good if they change the composition of this contributing set. Thus, the usual practice of assuming interior solutions in doing comparative statics is, in this case, quite misleading. An appropriate analysis must involve careful consideration of the boundary cases as well.

However, that analysis turns out to be possible, and indeed it yields quite strong results. We can use this analysis to illustrated the workings of our model in some interesting special cases. For example, we use our results to analyze the extent to which government provision of a public good will 'crowd out' private contributions and to demonstrate that 'equalizing' income redistributions tend to reduce the voluntary provision of a public good. Our neutrality theorems and other comparative static results provide a number of

\footnotetext{
${ }^{3}$ Related insights are to be found in Becker (1981), Sugden (1982), and Cornes and Sandler (1985).
} 
testable implications of the Nash hypothesis concerning the voluntary provision of public goods. This should help economists to develop new methods using both experimental methods and traditional empirical data to test the Nash hypothesis.

\section{A neutrality theorem}

Consider a simple model where there is one public good, one private good, and $n$ consumers. Each consumer $i$ consumes an amount $x_{i}$ of the private good and donates an amount $g_{i} \geqq 0$ to the supply of the public good. The total supply of public good, $G$, is just the sum of the gifts of all individuals. The utility function of consumer $i$ is $u_{i}\left(x_{i}, G\right)$. Consumer $i$ is endowed with wealth $w_{i}$ which he allocates between the private good $x_{i}$ and his gift $g_{i}$. We let $G_{-i}$ denote the sum of all gifts by consumers other than $i$ and make the Nash assumption that each consumer believes that the contributions of others are independent of his own.

Definition A Nash equilibrium in this model is a vector of gifts $\left(g_{i}^{*}\right)$, $i=1, \ldots, n$, such that for each $i,\left(x_{i}^{*}, g_{i}^{*}\right)$ solves

$$
\begin{array}{cl}
\max _{x_{i}, g_{i}} u_{i}\left(x_{i}, g_{i}+G_{-i}^{*}\right) \\
\text { s.t. } \quad x_{i}+g_{i}=w_{i} \\
\quad g_{i} \geqq 0 .
\end{array}
$$

Let us consider the maximization problem of a given consumer. Implicitly each consumer is choosing not only his gift, but in fact the equilibrium level of $G$ itself. For a consumer can decide to make a zero gift, in which case he chooses $G=G_{-i}$, or he can choose to make $G$ larger than $G_{-i}$. Thus, the consumer's maximization problem can also be written as

$$
\begin{array}{ll}
\max _{x_{i}, G} & u_{i}\left(x_{i}, G\right) \\
\text { s.t. } & x_{i}+G=w_{i}+G_{-1}^{*} \\
& G \geqq G_{-i}^{*} .
\end{array}
$$

This is just like an ordinary consumer choice problem except for the inequality constraint. We depict the equilibrium for a contributing consumer $i$ in a standard consumer theory diagram in fig. 1 . The budget constraint requires him to choose a point on the line $A D$ while the incquality constraint 
public good

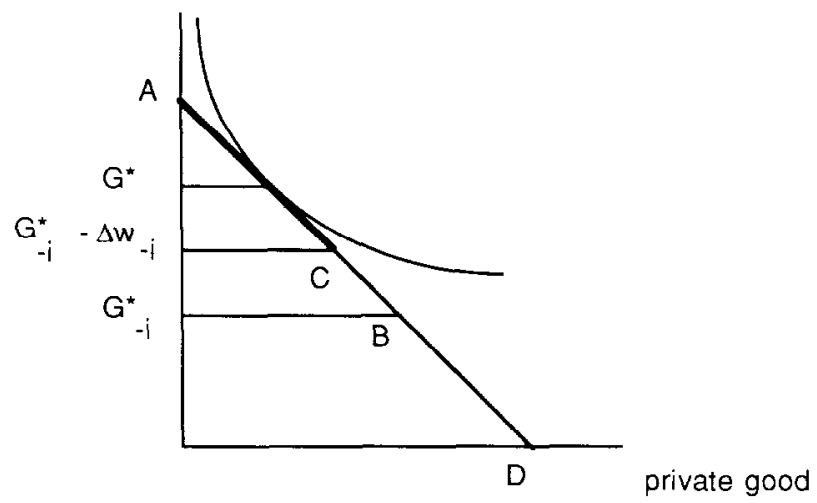

Fig. 1. Negative wealth transfer to consumer $i$.

restricts him to the segment $A B$ of that line. Fig. 3 depicts a case with three different consumers who all have the same preferences but different wealth levels. In the example depicted there, the two wealthiest consumers are contributors, while the third consumer chooses not to contribute.

Using a direct proof which fully describes the effect of an income redistribution on individual contributions, we are able to place exact boundaries on the class of redistributions that leave the set of contributors unchanged. ${ }^{4}$ Because of its directness, this proof allows us to see the intuition behind the theorem in a way that Warr's calculus proof does not.

Theorem 1. Assume that consumers have convex preferences and that contributions are originally in a Nash equilibrium. Consider a redistribution of income among contributing consumers such that no consumer loses more income than his original contribution. After the redistribution there is a new Nash equilibrium in which every consumer changes the amount of his gift by precisely the change in his income. In this new equilibrium, each consumer consumes the same amount of the public good and the private good that he did before the redistribution.

Proof. Suppose that in the original equilibrium $G^{*}$ is the total amount of contributions, $g_{i}^{*}$ is consumer $i$ 's contribution, and $\Delta w_{i}$ is the change in $i$ 's wealth caused by the redistribution. Suppose also that after the redistribution, every consumer other than consumer $i$ changes his contribution by the exact amount of his change in wealth. Since the changes in wealth of all contributors add to zero, the total change in the contributions of others will

${ }^{4}$ Cornes and Sandler (1985) use a similar type of proof. 
equal minus the change in consumer i's wealth. Therefore the budget equation for consumer $i$ does not change. However, the inequality requiring that gifts are non-negative becomes $G \geqq G_{-i}^{*}-\Delta w_{i}$, rather than $G \geqq G_{-i}^{*}$ as in the old equilibrium. Since, by assumption, $g_{i}^{*}+\Delta w_{i} \geqq 0$, the consumer can still obtain the old level $G^{*}$ of the public good and his old level of private consumption by simply changing his gift to $g_{i}^{*}+\Delta w_{i}$.

In fact, this is the best that he can do. We see this by considering two possible cases. If $\Delta w_{i}<0$, then his budget set is smaller than it was before the redistribution. This situation is depicted in fig. 1 , where the new budget set is the line segment $A C$. Since he can still afford the old bundle, he will, by the principle of revealed preference, choose it. The other possibility is that $\Delta w_{i}>0$. In this case the inequality constraint allows the consumer a larger segment of the budget line. The situation is as depicted in fig. 2, where the choice set is expanded from the line segment $A C$ to the line segment $A B$. Although the budget set is larger, if the consumer has convex preferences, the old bundle is at least as good as any of the new possibilities made available to him. This is true because if there were a better choice for him in the new segment, then there would have to be a convex combination of this preferred choice and the old choice which would be better than the old choice and attainable in the old situation. ${ }^{5}$ This establishes the theorem since we have shown that cach consumcr will choose to change his contribution by the change in his income if all other consumers do so. Q.E.D.

Note that in the new equilibrium each consumer has precisely the same consumption of the private and the public good as he had before. The

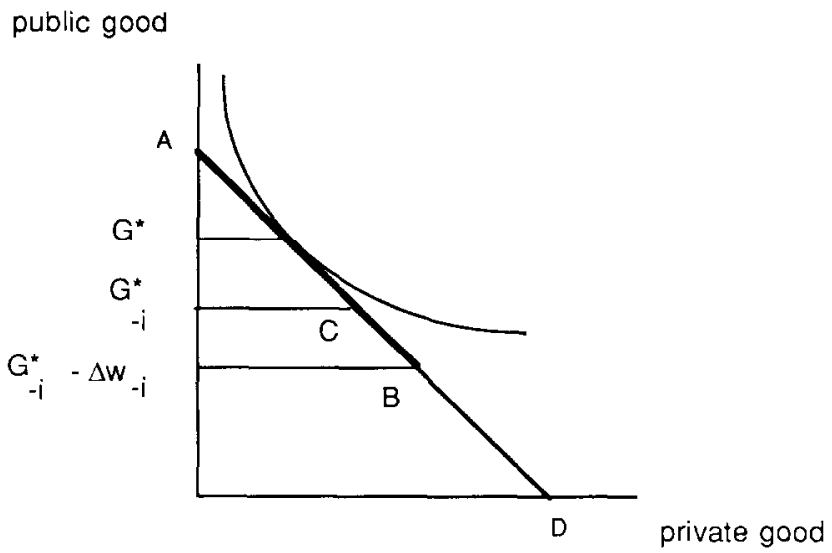

Fig. 2. Positive wealth transfer to consumer $i$.

\footnotetext{
${ }^{5}$ Notice that the convexity assumption is needed, since otherwise fig. 2 could be redrawn so that the new situation allows a choice preferred to the old choice.
} 
optimal responses of the consumers to the wealth transfer have completely offset the effects of the redistribution.

Before we turn to other comparative statics results, let us mention a few extensions of this result. First, there is no loss of generality in restricting ourselves to a public good which is a sum of the individual contributions. If instead $G=f\left(\sum_{i=1}^{n} g_{i}\right)$, utility would take the form $u_{i}\left(x_{i}, f(G)\right)$ which is still of the appropriate form. One slight extension of this form that is of particular interest is the form $u_{i}\left(x_{i}+f_{i}(G)\right)$. This sort of structure might be appropriate for modelling lobbying behavior: each agent contributes some amount $g_{i}$ to a lobbying fund which will then result in a payment to him of $f_{i}(G)$.

Secondly, it is possible to extend the result to more general solution concepts than that of Nash equilibrium. Suppose, for example, that each individual believes that when he decides to increase his gift by some amount $\mathrm{d} g_{i}$ he induces the other agents to change their aggregate contributions by $\mathrm{d} G_{-i}$. Furthermore, we will suppose that this 'conjectural variation' of individual $i$ is some function of $x_{i}$ and $G$ :

$$
\frac{\mathrm{d} G_{-i}}{\mathrm{~d} g_{i}}=h_{i}\left(x_{i}, G\right) \text {. }
$$

Then the first-order condition for the optimal gift by agent $i$ is simply:

$$
-\frac{\partial u_{i}\left(x_{i}, G\right)}{\partial x_{i}}+\frac{\partial u_{i}\left(x_{i}, G\right)}{\partial G}\left(1+h_{i}\left(x_{i}, G\right)\right)=0 .
$$

Now simply note that if each contributor changes his contribution by the amount of his wealth change $\Delta w_{i}$, this equation will still be satisfied, since neither $x_{i}$ or $G$ will change.

Furthermore, the neutrality result does not depend on there being only one public good. A similar argument establishes a neutrality result for the case of multiple public goods, as we show in Theorem 7 .

However, the result is sensitive to the assumption that utility depends only on private consumption and the amount of the public good. If utility also depended on the amount of agent $i$ 's gift for example, the result would not go through in general, although it does work for some special forms of utility. For example, if the preferences between $x_{i}, g_{i}$, and $G$ were quasihomothetic the equilibrium demand for $g_{i}$ would have the form $g_{i}=a_{i}(G)$ $+b(G) w_{i}$. It is easy to see that Warr's neutrality result will still obtain. However, the quasihomothetic preference structure is quite restrictive. Whether there are less restrictive preference structures that give rise to the same neutrality result is an open question, although the results of Bergstrom and Varian (1984) indicate that the linearity of demand in wealth is certainly a necessary condition. 


\section{Existence, uniqueness, and comparative statics}

The neutrality result in the previous section only applies when the redistribution of wealth is sufficiently small so as not to change the set of contributors. But in many interesting examples one cares about redistributions of arbitrary size, and the change in the set of contributors is of significant interest.

In order to study the existence and uniqueness of a Nash cquilibrium in voluntary contributions and to develop some comparative statics results for arbitrary redistributions, we find it useful to study Nash 'reaction functions'. Suppose that the contributions of others add up to $G_{-i}$. From our remarks in the previous section it follows that consumer $i$ will choose his own contribution $g_{i}$ so that $G=G_{-i}+g_{i}$ solves the following constrained maximization problem:

$$
\begin{aligned}
& \max _{x_{i}, G} u_{i}\left(x_{i}, G\right) \\
& \text { s.t. } \quad x_{i}+G=w_{i}+G_{-i}^{*} \\
& \quad G \geqq G_{-i}^{*} .
\end{aligned}
$$

Ignoring the inequality constraint, this is formally the same as a standard demand problem for a consumer with income $w_{i}+G_{-i}^{*}$. Let $f_{i}(w)$ be consumer $i$ 's demand function for the public good, representing the value of $G$ that $i$ would choose as a function of the right-hand side of the above budget constraint, ignoring the inequality constraint. Then his demand for the public good, taking the inequality constraint into account, is simply:

$$
G=\max \left\{f_{i}\left(w_{i}+G_{-i}\right), G_{-i}\right\}
$$

Subtracting $G_{-i}$ from each side of this equation we have individual $i$ 's optimal response:

$$
g_{i}=\max \left\{f_{i}\left(w_{i}+G_{-i}\right)-G_{-i}, 0\right\} .
$$

In the previous section we made no assumptions on preferences other than strict convexity. In this section our results depend on the following assumption on the demand functions for the public good.

Assumption There is a single-valued demand function for the public good, $f_{i}(w)$, which is a differentiable function of wealth. The marginal propensity to consume the public good is greater than zero and less than 1 so that $0<f_{i}^{\prime}(w)<1$ for all $i=1, \ldots, n$. 
The assumption that the marginal propensity to consume the public good is between zero and one scems innocuous. It simply requires that both the public and the private good be normal goods for all consumers. While this assumption is not needed for existence, it will be needed for uniqueness and it greatly simplifies our later comparative statics analyses.

Theorem 2. A Nash equilibrium exists.

Proof. Let $W=\left\{x\right.$ in $R^{n}: 0 \leqq x_{i} \leqq w_{i}$ for $\left.i=1, \ldots, n\right\}$. This is clearly a compact and convex set. The functions $g_{i}=\max \left\{f_{i}\left(w_{i}+G_{-i}\right)-G_{-i}, 0\right\}$ define a continuous function from the set $W$ to itself. Hence, by Brouwer's Fixed Point Theorem there must exist a fixed point, which is a Nash equilbrium vector of gifts. Q.E.D.

Let $C$ denote the set of consumers that are actually contributing in some equilibrium; i.e. $g_{i}>0$ if and only if $i$ is in $C$. From eq. (1) the following useful result is immediate.

Fact 1. A configuration of gifts is a Nash equilibrium if and only if the following conditions are satisfied:

$$
\begin{aligned}
& G=f_{i}\left(w_{i}+G_{-i}\right) \text { for } i \text { in } C, \\
& G \geqq f_{j}\left(w_{j}+G_{-j}\right) \text { for } j \text { not in } C .
\end{aligned}
$$

We can now provide a simple characterization of an equilibrium that will be useful for comparative statics results.

Fact 2. There exists a real valued function $F(G, C)$, differentiable and increasing in $G$, such that in a Nash equilibrium:

$$
F(G, C)=\sum_{i \in C} w_{i}
$$

Prooff. Since $f_{i}(w)$ is a strictly increasing function of wealth for each individual, it has an inverse, $\phi_{i}$. Applying $\phi_{i}$ to each side of (2) we have:

$$
\phi_{i}(G)=w_{i}+G_{-i} \text { for } i \in C
$$

Summing these equations and rearranging we have:

$$
\sum_{i \in C} \phi_{i}(G)+(1-c) G=\sum_{i \in C} w_{i}
$$


where $c$ is the number of contributing consumers. Letting $F(G, C)$ denote the left-hand side of this relationship, we have the desired cquation. To show that $F(G, C)$ is increasing in $G$, we note that since $f_{i}^{\prime}(w)<1, \phi_{i}^{\prime}(G)>1$. Differentiating $F(G, C)$ with respect to $G$ gives:

$$
\partial F(G, C) / \partial G=\sum_{i \in C} \phi_{i}^{\prime}(G)+(1-c)>c+(1-c)=1>0 .
$$

Hence, $F(G, C)$ is a strictly increasing function of $G$. Q.E.D.

Theorem 3. There is a unique Nash equilibrium with a unique quantity of public good and a unique set of contributing consumers.

Proof. Consider two Nash equilibria in which the sets of contributing consumers are $C$ and $C^{\prime}$ and the total amounts of the public good supplied are $G$ and $G^{\prime}$. Without loss of generality, assume that $G^{\prime} \leqq G$. Applying $\phi_{i}$ to both sides of (2) and (3), we see that

$$
w_{i}+G_{-i}^{\prime} \leqq \phi_{i}\left(G^{\prime}\right)
$$

for all $i$ in $C$. Summing these equations and rearranging terms, we have:

$$
\sum_{i \in C} w_{i} \leqq F\left(G^{\prime}, C\right)
$$

But fact 2 informs us that:

$$
\sum_{i \in \mathbf{C}} w_{i}=F(G, C)
$$

Since $F$ is monotone increasing in $G$, it follows that $G^{\prime} \geqq G$. But, by construction, $G^{\prime} \leqq G$. Therefore $G^{\prime}=G$ so that the Nash equilibrium quantity of the public good is uniquely determined. From (3) we see that the set of non-contributors is uniquely determined by $G$. Since $G^{\prime}=G$, it follows that $C^{\prime}=$ C. Q.E.D.

The above analysis suggests a way to calculate an equilibrium. Start by choosing an arbitrary subset of consumers $C$. Eq. (4) has a unique solution $G$, and given $G$, we can solve (2) for a unique $g_{i}$ for $i=1, \ldots, n$. If (3) is satisfied, we are done. If not, we choose a different set $C$. Since there is only a finite number of subsets $C$, and, according to Theorem 3 , a unique equilibrium exists, this procedure will eventually terminate.

It is immediate from fact 2 that a redistribution of wealth that does not change the composition of the contributing set will increase, decrease, or leave unchanged the equilibrium amount of the public good as it increases, 
decreases, or leaves unchanged the aggregate wealth of the contributing set. However, 'large' redistributions of wealth will typically change the composition of the contributing set. Nevertheless, we are able to establish some strong comparative statics results that apply to general changes in the income distribution. An important tool for establishing these results is the following.

Fact 3. Let $\left(g_{i}\right)$ and $\left(g_{i}^{\prime}\right) i=1, \ldots, n$ be Nash equilibria given the wealth distributions $\left(w_{i}\right)$ and $\left(w_{i}^{\prime}\right)$, let $C$ and $C^{\prime}$ be the corresponding sets of contributing consumers, and let the function $F(G, C)$ be as defined in fact 2 . Then

$$
F\left(G^{\prime}, C\right)-F(G, C) \geqq \sum_{i \in C}\left(w_{i}^{\prime}-w_{i}\right)
$$

Proof. From fact 1 it follows that $f_{i}\left(w_{i}^{\prime}+G_{-i}^{\prime}\right) \leqq G^{\prime}$ for all $i$ in $C$. Therefore, applying $\phi=f^{-1}$,

$$
w_{i}^{\prime}+G_{-i}^{\prime} \leqq \phi_{i}\left(G^{\prime}\right)
$$

for all $i$ in $C$. Summing these inequalities over all $i$ in $C$, and rearranging terms we have:

$$
\sum_{i \in C} w_{i}^{\prime} \leqq \sum_{i \in C} \phi_{i}\left(G^{\prime}\right)-c G^{\prime}+\sum_{i \in C} g_{i}^{\prime}
$$

Since $\sum_{i \in C} g_{i}^{\prime} \leqq G^{\prime}$, it follows that

$$
\sum_{i \in C} w_{i}^{\prime} \leqq F\left(G^{\prime}, C\right)
$$

But, according to fact 2,

$$
\sum_{i \in C} w_{i}=F(G, C)
$$

Therefore

$$
F\left(G^{\prime}, C\right)-F(G, C) \geqq \sum_{i \in C}\left(w_{i}^{\prime}-w_{i}\right) . \quad \text { Q.E.D. }
$$

Theorem 4 collects a number of comparative statics results that follow directly from fact 3 .

Theorem 4. In a Nash equilibrium:

(i) Any change in the wealth distribution that leaves unchanged the aggregate 
wealth of current contributors will either increase or leave unchanged the equilibrium supply of public good.

(ii) Any change in the wealth distribution that increases the aggregate wealth of current contributors will necessarily increase the equilibrium supply of the public good.

(iii) If a redistribution of income among current contributors increases the equilibrium supply of the public good, then the set of contributing consumers after the redistribution must be a proper subset of the original set of contributors.

(iv) Any simple transfer of income from another consumer to a currently contributing consumer will either increase or leave constant the equilibrium supply of the public good.

Proof. According to fact 3 , if $\sum_{i \in C}\left(w_{i}^{\prime}-w_{i}\right)=0$, then $F\left(G^{\prime}, C\right) \geqq F(G, C)$. Since, according to fact $2, F(G, C)$ is increasing in $G$, it must be that $G^{\prime} \geqq G$. This proves assertion (i). Similarly, it follows from fact 3 that if $\sum_{i \in C}\left(w_{i}^{\prime}-w_{i}\right)>0$, then $F\left(G^{\prime}, C\right)>F(G, C)$ and therefore $G^{\prime}>G$. This proves assertion (ii).

Suppose that the income distribution $\left(w_{i}^{\prime}\right), i=1, \ldots, n$, is obtained from the distribution $\left(w_{i}\right), i=1, \ldots, n$, by a redistribution of income among the members of $C$. If $G^{\prime}>G$, then it follows from fact 1 that consumers who contributed nothing before the redistribution will contribute nothing after. Therefore $C^{\prime} \subset C$. If $C^{\prime}=C$, then the change from the income distribution $\left(w_{i}^{\prime}\right)$ to the distribution $\left(w_{i}\right)$ leaves the total income of contributors in $C^{\prime}$ unchanged. Therefore according to assertion (i) it must bc that $G \geqq G^{\prime}$. But this is impossible if $G^{\prime}>G$. It follows that if $G^{\prime}>G$, then $C^{\prime}$ is a proper subset of $C$. This proves assertion (iii).

Since any simple transfer of income to a current contributor from some other consumer (whether that consumer is a contributor or not) will either increase or leave constant the aggregate wealth of current contributors, assertion (iv) is immediate from assertions (i) and (ii). Q.E.D.

While it is true that a transfer of wealth from non-contributors to contributors will increase total contributions, it is not true that a transfer of income from contributors to non-contributors will necessarily decrease contributions, since some of the non-contributors may then decide to begin contributing. However, Theorem 4 establishes that a transfer in the other direction - from non-contributors to contributors - will necessarily increase the supply of the public good, even if the set of contributors changes. The difference between assertion (i) of Theorem 4 and the neutrality result of Theorem 1 is that assertion (i) applies even to redistributions in which some contributors may lose more income than they are currently giving. For such a redistribution, the total amount of gifts may increase even though total income of the original set of consumers is constant and no new contributors 
are added. But, as assertion (iii) maintains, this will occur only if the redistribution reduces the size of the set of contributing consumers.

\section{Equilibrium with identical tastes}

Equilibrium is even more simply characterized if all consumers have the same demand function for the public good. In this case there is some critical wealth level such that consumers with wealth greater than that level are contributors, and consumers with wealth less than that level are not contributors. Furthermore, we can show that when consumers are identical, the more equal the wealth distribution, the less of the public good will be supplied. To make this result precise, we need the following definition.

Definition. A redistribution of wealth is said to be equalizing if it is equivalent to a series of bilateral transfers in which the absolute value of the wealth difference between the two parties to the transfer is reduced.

Let $f(\cdot)$ be the demand function, which is identical for all consumers, and let $\phi(\cdot)$ be the inverse function of $f(\cdot)$. Then we have the following useful characterization of equilibrium.

Fact 4. If all consumers have identical preferences and $G^{*}$ is an equilibrium supply of the public good, then there is a critical wealth level $w^{*}=\phi\left(G^{*}\right)-G^{*}$ such that all consumers with wealth $w_{i} \leqq w^{*}$ contribute nothing and every consumer with income $w_{i}>w^{*}$ contributes the amounts $g_{i}^{*}=w_{i}-w^{*}$ to the supply of the public good.

Proof. In equilibrium, according to fact 1 , for all $i, f\left(w_{i}+G_{-i}^{*}\right) \leqq G^{*}$ with equality if $g_{i}^{*}>0$. Therefore

$$
w_{i}+G_{-i}^{*} \leqq \phi\left(G^{*}\right) \text { with equality if } g_{i}^{*}>0
$$

But $G_{-i}^{*}=G^{*}-g_{i}^{*}$, so that we can rewrite the previous inequality as

$$
w_{i}-g_{i}^{*} \leqq \phi\left(G^{*}\right)-G^{*} \text { with equality if } g_{i}^{*}>0 .
$$

Let $w^{*}=\phi\left(G^{*}\right)-G^{*}$. Then the previous inequality implies that:

$$
g_{i}^{*}=w_{i}-w^{*} \quad \text { if } w_{i}>w_{i}^{*} \quad \text { and } \quad g_{i}^{*}=0 \quad \text { if } w_{i} \leqq w^{*} \text {. Q.E.D. }
$$

Armed with fact 4 , we can demonstrate the following additional properties of equilibrium. 
Theorem 5. If preferences are identical, then in a Nash equilibrium:

(i) All contributors will have greater wealth than all non-contributors.

(ii) All contributors will consume the same amount of the private good as well as of the public good.

(iii) An equalizing wealth redistribution will never increase the voluntary equilibrium supply of the public good.

(iv) Equalizing wealth redistributions among current non-contributors or among current contributors will leave the equilibrium supply unchanged.

(v) Equalizing income redistributions that involve any transfers from contributors to non-contributors will decrease the equilibrium supply of the public good.

Proof. Assertions (i) and (ii) are immediate from fact 4. To prove assertion (iv), let $G^{*}$ be the initial equilibrium supply and let $w^{*}=\phi\left(G^{*}\right)-G^{*}$. An equalizing wealth redistribution between two consumers with wealths smaller than $w^{*}$ will leave them both with wealths below $w^{*}$ after the transfer. Therefore $G^{*}$ will remain an equilibrium with the same set of contributors as in the initial equilibrium. An equalizing wealth redistribution between two consumers with wealths at least as large as $w^{*}$ will leave them both with wealths at least as large as $w^{*}$. It follows from fact 4 that neither consumer loses more wealth than his contributions in the initial equilibrium. Therefore it follows from Theorem 1 that $G^{*}$ remains an equilibrium. Since all equalizing distributions that do not involve a transfer from a contributing consumer to a non-contributing consumer must be made up of a sequence of transfers of this type, redistributions of this type will leave the equilibrium supply unchanged.

To prove assertion (v), consider an equalizing redistribution between two consumers in which wealth is distributed from some consumer $i$ with $w_{i}>w^{*}$ to a consumer $j$ with $w_{j}<w^{*}$. In this case we claim that equilibrium provision of the public good must fall. For suppose the new equilibrium provision is $G^{\prime} \geqq G^{*}$. Then the new threshold income is $w^{\prime}=\phi\left(G^{\prime}\right)-G^{\prime}$ and $w^{\prime} \geqq w^{*}$. From fact 4 it then follows that those consumers whose wealths did not change would not increase but might decrease their contributions. Let $\Delta$ be the amount of wealth that is transferred from $i$ to $j$. If $w_{i}-\Delta>w^{\prime}$ and $w_{j}+\Delta>w^{\prime}$, then from fact 4 and the fact that $w^{\prime} \geqq w^{*}$ it follows that consumer $i$ will diminish his contribution by at least $\Delta$ and consumer $j$ will increase his contribution by less than $\Delta$. If after the redistribution only one of the two consumers $i$ and $j$ has income greater than $w^{\prime}$, then let $w^{+}$be the larger of $w_{i}^{\prime}$ and $w_{j}^{\prime}$. Then the total contributions of $i$ and $j$ after the redistribution will be $w^{+}-w^{\prime}$. Since the redistribution was an equalizing redistribution, it must be that $w^{+}<w_{i}$. Therefore $w^{+}-w^{\prime}<w_{i}-w^{*}$. But total contributions by $i$ and $j$ before the redistribution were just $w_{i}-w^{*}$. It follows that for all bilateral redistributions from a contributor $i$ to a non-contributor $j$, total contri- 
butions of $i$ and $j$ will diminish while contributions of all other consumers will not increase. Therefore total contributions must decrease. But this implies that $G^{\prime}<G^{*}$, which is contrary to our hypothesis that $G^{\prime} \geqq G^{*}$. Since any equalizing transfer that involves a transfer from some contributor to some non-contributor can be constructed as a series of bilateral transfers of this type of the type covered in assertion (iv), we have a proof of assertion (v). Assertion (iii) is immediate from (iv) and (v). Q.E.D.

The surprising result that all contributors have the same public and private consumption also has a simple geometric proof. Consider fig. 3 , and assume that all consumers have identical preferences so that the indifference curves depicted there come from a single preference ordering. Suppose, as shown, that agents 2 and 3 have different wealths and different privatc consumption. Then increasing agent 2's wealth to the level of agent 1 would result in no change in the demand for the public good $G$, violating our assumption of strict normality. This contradiction establishes the result.

Theorem 5 can be extended in the following way to an economy in which preferences are not identical. Suppose that there are a number of different 'types' of consumers. With minor modifications of our proof, we can demonstrate that all consumers of the same type who contribute to the public good must have the same private consumption. Where an 'equalizing redistribution' is defined as one which consists of a series of equalizing transfers between consumers of the same type, we can also show that the conclusions of Theorem 5 hold.

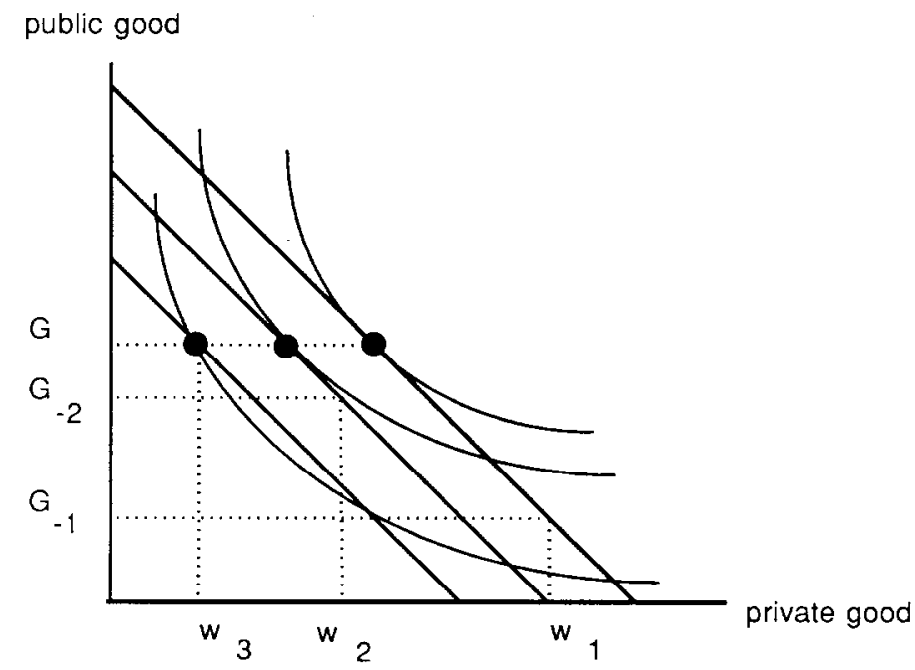

Fig. 3. A typical Nash equilibriun. 
If preferences are homothetic as well as identical, we can describe equilibrium even more explicitly. In this case the demand functions have the special form $f(w)=\alpha w$. Let us order the consumers so that $w_{1} \geqq w_{2} \geqq \cdots \geqq w_{n}$. One possible equilibrium would have the richest person be the sole contributor. Then his contributions would be $f\left(w_{1}\right)=\alpha w_{1}$. The second wealthiest person will not contribute if

$$
G=\alpha w_{1} \geqq \alpha\left(w_{2}+G\right)=\alpha\left(w_{2}+\alpha w_{1}\right),
$$

which reduces to

$$
(1-\alpha) w_{1} \geqq w_{2}
$$

Thus, if $\alpha=0.5$, and the richest individual is more than twice as wealthy as the second richest, he will be the only contributor to the public good.

Another interesting case is where the wealthiest individual has all of the wealth $W$ and everyone else has zero wealth. In this case the wealthiest individual chooses $G_{1}=\alpha W$ which is easily seen to be a Pareto-efficient level of the public good. Now divide his wealth between two individuals. Theorem 5 implies that they must both be equal contributors to the public good since they have the same wealth. Therefore

$$
G_{2}=\alpha\left(W / 2+G_{2} / 2\right)
$$

or equivalently,

$$
G_{2}=\alpha W /(2-\alpha),
$$

which is strictly less than $G_{1}$ since $\alpha$ is less than 1 . More generally, if all wealth is divided equally among the $k$ consumers, we have:

$$
G_{k}-\alpha W /(k-\alpha)
$$

so the level of the public good diminishes as the income distribution becomes more equal. The smallest amount of the public good is supplied when everyone is a contributor.

A further case where one can get explicit solutions is the case of quasilinear utility, i.e. where the utility function has the form:

$$
u_{i}\left(x_{i}, G\right)=x_{i}+h_{i}(G)
$$

Let us take the particular example of

$$
u_{i}\left(x_{i}, G\right)=x_{i}+a_{i} \ln G .
$$


There are two interesting cases. First, suppose that $a_{i}=1$ for all $i=1, \ldots, n$. It is possible to show that any set of gifts $\left(g_{i}\right), i=1, \ldots, n$, such that $0 \leqq g_{i} \leqq 1$ and $\sum_{i=1}^{n} g_{i}=1$ is an equilibrium. ${ }^{6}$

Now suppose that $a_{1}=1$ and $a_{i}<1$ for $i=2, \ldots, n$. In this case the unique equilibrium is $g_{1}=G=1$ and $g_{i}=0$ for $i=2, \ldots n$. In this equilibrium person 1 contributes the entire amount of the public good, and all the other agents free ride! Thus, a very small change in the preference can lead to a large change in the structure of the equilibrium. This example shows how special the case of symmetric equilibria is when free rider problems are present.

\section{The effect of government supply on private donations}

Suppose that the government provides some quantity of public goods which it pays for with tax revenues. Could it be that the government provision 'crowds out' an equal amount of private donations? Abrams and Schmitz (1978) pose this question and attempt to answer it empirically. Using time series data for the United States, they regress private charitable donations on government expenditures on health, hospitals, education, and welfare. Their estimates 'indicate a crowding out effect on the order of 28 per cent. This implies that a one dollar increase in governmental transfers lowers private charitable contributions by approximately 28 cents'. Roberts (1984) cites historical evidence that the introduction of large-scale government welfare programs in the United States was accompanied by a reduction in private charitable contributions. Roberts' figures also show that the total amount of relief funds, private and public, rose during this period. In the context of a somewhat different model, Brennan and Pincus (1983) suggest that when public expenditures are supplemented by private contributions, small increases in public contributions are likely to displace equal amounts of private contributions.

Warr (1982) and Roberts (1984) present theoretical analyses which yield the rather startling result that government contributions result in a dollar for dollar' reduction in private contributions. Their results rely on the assumption that all of the taxes that pay for the government's contribution are collected from contributors. Typically, we would argue, that in equilibrium, the set of contributing consumers is only a small subset of the taxpaying population. Therefore the cxtra taxes paid by contributing consumers are likely to be much less than the government's contribution. Theorem 6 shows that under these circumstances the crowding out effect of government contributions will be only partial.

\footnotetext{
${ }^{6}$ Note that this example does not violate our uniqueness result since $G$ is not a strictly normal good when utility is quasilinear.
} 
Theorem 6. Suppose that starting from an initial position where consumers supply a public good voluntarily, the government supplies some amount of the public good which it pays for from tax's. Then:

(i) If the taxes collected from each indiridual do not exceed his voluntary contribution to the public good in the absence of government supply, then the government's contribution results in an equal reduction in the amount of private contributions.

(ii) If the government collects some of the taxes that pay for its contribution from non-contributors, then, although private contributions may decrease, the equilibrium total supply of the public good must increase.

(iii) If the government collects some of the taxes that pay for its contribution by taxing any contributor by more than the amount of his contribution, the equilibrium total supply of the public good must increase.

Proof. Suppose that the government collects taxes $t_{i}$ from each consumer $i$ and supplies an amount $g_{0}$ of the public good, where $\sum_{i=1}^{n} t_{i}=g_{0}$. The total amount of the public good supplied will be $G=g_{0}+\sum_{i=1}^{n} g_{i}$. Let $G_{-i}=$ $G-g_{i}$. The budget constraint of consumer $i$ can be written $x_{i}+g_{i}=w_{i}-t_{i}$ or, equivalently, $x_{i}+G=w_{i}-t_{i}+G_{-i}$. By the same reasoning that established fact 2, we see that in Nash equilibrium it must be that conditions (2) and (3) apply in this model as well. Defining the function $\phi_{i}$ as in the proof of fact 2, we have, as before, in Nash equilibrium, $\phi_{i}(G)=w_{i}-t_{i}+G_{-i}$ for all $i$ in $C$. Summing these equations over all $i$ in $C$, we find:

$$
F(G, C)=\sum_{i \in C} w_{i}-\sum_{i \in C} t_{i}+g_{0}
$$

where $F(G, C)$ is defined as in fact 2. Therefore government provision of the public good has exactly the same effect as a wealth transfer in which the total wealth of the set of contributing consumers is changed by $g_{0}-\sum_{i \in C} t_{i}$. If all consumers are contributors, then the taxes collected from contributors must necessarily equal the total government contributions so that $g_{0}-\sum_{i \in c} t_{i}$ $=0$. From Theorem 1 it then follows that the equilibrium supply of the public good remains constant despite the government provision of the good. This proves assertion (i). But if the government collects some taxes from noncontributors, then $g_{0}-\sum_{i \in C} t_{i}>0$. This is then equivalent to a transfer that increases the wealth of the set of contributors, which, by part (ii) of Theorem 4 , must increase the equilibrium supply of the public good. This proves assertion (ii).

To prove part (iii), we suppose that some consumer is taxed by more than his voluntary contribution. Think of this as first taxing the consumer by the exact amount of his contribution, then taxing him for the extra amount. The first step leaves the amount of the public good unchanged by assertion (i), 
and the second step increases the amount of the public good by assertion (ii). Q.E.D.

Our Theorem 6 is certainly consistent with the findings of Abrams and Schmitz and of Roberts that there is 'partial crowding out'. However, it would be interesting to extend the empirical explorations begun by these authors to try to determine whether some of the reduction in private donations which they attribute to crowding out by public expenditures can be explained by a reduction in income inequality. It would also be interesting to look at cross-section data across different countries or localities in addition to the single country time series that they have examined. Theorems 5 and 6 also pose a number of strong testable hypotheses that could be investigated by experimental economists who could, for example, impose identical payoff functions, or could introduce entirely exogenous changes in the amount of public goods supplied and taxes collected by a 'government'.

Brennan and Pincus (1983) suggest that much of the growth of government expenditures in industrialized countries in the twentieth century must be accounted for, not by an increase in demand for public goods, but rather by the fact that government provision of goods crowds out private expenditures on the same goods so that the 'effects of enormous increases in government spending on the aggregate pattern of consumption in the economy ... are probably quite small'. This argument is buttressed by the empirical work of Borcherding (1977), who shows that in the United States in the twentieth century, state and local government expenditures have risen much more rapidly than can be explained by changes in income, price, and other standard economic variables.

It would be nice to have an explanation not only of what happens to private contributions when the government increases its contribution of a public good, but also of what causes the government to do so. Our results offer a clue that may provide a partial explanation for the historical increase in government's role. In the model that we treat, private contributions will in general supply less than a Pareto-optimal amount of public goods. On the other hand, we have not incorporated the inefficiencies of administration, the informational imperfections inherent in demoncratic decision-making, and the excess burden involved in collecting taxes. These effects, if properly accounted for, could suggest good reasons why private rather than public provision would sometimes be efficient. As Theorem 5 indicates, the amount supplied by voluntary contributions will tend to be smaller the more equally income is distributed. Thus, if an economy evolves toward a more equal distribution of income we can expect the amount of public goods that would be provided voluntarily to diminish. This means that the case for government provision in the interest of efficiency would become stronger as the income distribution becomes more equal and might eventually overcome the advantages of private provision. 


\section{An extension to several public goods}

Theorem 1 generalizes in an interesting way to the case of several public goods. Let us suppose that in equilibrium any consumer can contribute any non-negative amount that he chooses to the supply of any of the public goods. For economy of exposition, we will confine our formal discussion to the case of two public goods, $G$ and $H$. All of our discussion extends in a fairly obvious way to higher dimensions. For the purposes of this section we define a Nash equilibrium as follows.

Definition. A Nash equilibrium is a vector of gifts $\left(g_{i}^{*}\right)$ and $\left(h_{i}^{*}\right), i=1, \ldots, n$, such that for each $i,\left(\mathrm{~g}_{i}^{*}, h_{i}^{*}\right)$ maximizes

$$
u_{i}\left(x_{i}, g_{i}+G_{-i}^{*}, h_{i}+H_{-i}^{*}\right)
$$

subject to the constraints:

$$
\begin{aligned}
& x_{i}+g_{i}+h_{i}=w_{i} \\
& g_{i} \geqq 0 \text { and } h_{i} \geqq 0
\end{aligned}
$$

The existence of such a Nash equilibrium is a simple generalization of Theorem 2. Now if the sets of donors to the two public goods are disjoint, we can use exactly the same argument that we gave in theorem 1 to establish a neutrality result - we can redistribute wealth among the $G$ contributors or among the $H$ contributors and we will get exactly the same kind of neutrality theorem. However, if the two sets of contributors are not disjoint, we may have difficulties. For in this case, redistributing wealth among the contributors to $G$ may change the wealth of the some of the contributors to $H$. This in turn may change their contributions to $H$ and thus change the equilibrium. As it turns out, we are able to show that if an income distribution among contributors leaves aggregate income of contributors to $H$ and of contributors to $G$ constant, then equilibrium total contributions are unchanged for each public good.

It is convenient to make the following definitions. Let GDONORS and HDONORS indicate the set of consumers who contribute to $G$ and $H$, respectively, and let GONLY and HONLY be the consumers that contribute only to $G$ and only to $H$. Let BOTH be the set of consumers that contribute positive amounts to both public goods, and DONORS the set of consumers that contribute positive amounts to either public good. The restriction that a redistribution among contributors leaves total income of contributors to $G$ and of contributors to $H$ constant is expressed as the following. 
Assumption $A$. The wealth redistribution among the donors satisfies:

$$
\sum_{i \in G D O N O R S} \Delta w_{i}=\sum_{i \in \text { HDONORS }} \Delta w_{i}=\sum_{i \in \text { DONORS }} \Delta w_{i}=0
$$

Although the following assumption may seem stronger, it is actually equivalent to Assumption A.

Assumption B. The wealth redistribution among the donors satisfies:

$$
\sum_{i \in G O N L Y} \Delta w_{i}=\sum_{i \in H O N L Y} \Delta w_{i}=\sum_{i \in B O T H} \Delta w_{i}=\sum_{i \in D O N O R S} \Delta w_{i}=0
$$

The proof that these assumptions are equivalent is a simple exercise in addition. We can now prove the following neutrality theorem.

Theorem 7. Let $u_{i}\left(x_{i}, G, H\right)$ be continuous and quasiconcave, and let $\left(x_{i}^{*}, g_{i}^{*}, h_{i}^{*}\right)$, for $i=1, \ldots, n$, be a Nash equilibrium. Let $\left(\Delta w_{i}\right), i=1, \ldots, n$, be a wealth transfer satisfying Assumption $A$ or $B$ and

$$
g_{i}^{*}+h_{i}^{*}+\Delta w_{i}>0 \text { for } i \in D O N O R S
$$

Then after the redistribution there is a Nash equilibrium in which the total supply of each public good and the private consumption of each consumer is the same as before the redistribution.

Proof. The argument is similar to that given in the proof of Theorem 1. Let us indicate the initial equilibrium by $\left(G^{*}, H^{*}\right)$. Given the wealth transfer $\left(A w_{i}\right)$ we can assume that each consumer in GONLY changes his contribution by $\Delta g_{i}=\Delta w_{i}$, each consumer in $H O N L Y$ changes his contribution by $\Delta h_{i}=\Delta w_{i}$, and that the consumers in BOTH change their contributions in the following way:

$$
\begin{aligned}
& \Delta g_{i}=\frac{G_{\text {BOTH }}^{*}}{G_{\text {BOTH }}^{*}+I I_{\text {BOTH }}^{*}}\left(g_{i}^{*}+h_{i}^{*}+\Delta w_{i}\right)=g_{i}^{*}, \\
& \Delta h_{i}=\frac{H_{\text {BOTH }}^{*}}{G_{\text {BOTH }}^{*}+H_{\text {BOTH }}^{*}}\left(g_{i}^{*}+h_{i}^{*}+\Delta w_{i}\right)-h_{i}^{*},
\end{aligned}
$$

where $G_{B O T H}^{*}=\sum_{i \in B O T H} g_{i}^{*}$ and $H_{B O T H}^{*}$ is defined in a similar manner.

This is clearly feasible, so we only need show that it is optimal for each consumer if the amount given by the others is $\left(G_{-i}^{*}-\Delta g_{i}, H_{-i}^{*}-\Delta h_{i}\right)$. Suppose not. Then there exists some new choice $\left(x_{i}^{\prime}, g_{i}^{\prime}, h_{i}^{\prime}\right)$ that consumer $i$ prefers to the choice $\left(x_{i}^{*}, g_{i}^{*}+\Delta g_{i}, h_{i}^{*}+\Delta h_{i}\right)$ and that is feasible for him. In 
symbols:

$$
\begin{aligned}
u_{i}\left(x_{i}^{\prime}, g_{i}^{\prime}\right. & \left.+G_{-i}^{*}-\Delta g_{i}, h_{i}^{\prime}+H_{-i}^{*}-\Delta h_{i}\right) \\
& >u_{i}\left(x_{i}^{*}, g_{i}^{*}+\Delta g_{i}+G_{-i}^{*}-\Delta g_{i}, h_{i}^{*}+\Delta h_{i}+H^{*}-\Delta h_{i}\right) .
\end{aligned}
$$

Since both the preferences and the budget set are convex, we can suppose that $\left(x_{i}^{\prime}, g_{i}^{\prime}, h_{i}^{\prime}\right)$ is arbitrarily close to $\left(x_{i}^{*}, g_{i}^{*}+\Delta g_{i}, h_{i}^{*}+\Delta h_{i}\right)$. If this is so, then $g_{i}^{\prime}+\Delta w_{i}>0$ for those consumers giving to $G$ and $h_{i}^{\prime}+\Delta w_{i}>0$ for those consumers giving to $H$. But then the choice $\left(x_{i}^{\prime}, g_{i}^{\prime}-\Delta g_{i}, h_{i}^{\prime}-\Delta h_{i}\right)$ would be feasible under the consumer's original budget constraint and rearranging the above inequality shows that it would be preferred to his original choice since:

$$
u_{i}\left(x_{i}^{\prime}, g_{i}^{\prime}-\Delta g_{i}+G_{-i}^{*}, h_{i}^{\prime}-\Delta h_{i}+H_{-i}^{*}\right)>u_{i}\left(x_{i}^{*}, g_{i}^{*}+G_{-i}^{*}, h_{i}^{*}+H_{-i}^{*}\right) \text {. }
$$

This contradiction establishes the result. Q.E.D.

Kemp (1984) proposes an extension of Warr's neutrality theorem to the case of several public goods. He argues, by counting equations and unknowns in the calculus first-order conditions, that if all consumers are making positive voluntary contributions to the supply of all public goods, then a small redistribution of income will have no effect on either the equilibrium supply of public goods or on any individual's consumption. Kemp's theorem shares with Warr's results the usual perils of assertions about the existence of a unique equilibrium on the grounds of equality of the numbers of equations and unknowns. ${ }^{7}$ A second weakness is that the Kemp proof makes essential use of the assumption that all consumers voluntarily contribute positive amounts of all public goods. This is a very strong assumption even if there is only one public good. ${ }^{8}$ In general we would expect that if consumers do not have identical preferences and incomes, it would be an extreme accident to find an equilibrium in which the set of contributors was the same for all public goods.

We are able to prove a theorem that does not require that everyone contributes to all public goods, but the result applies only to those redistributions that leave the total wealth of contributors to each public good unchanged, as well as leaving the total wealth constant for those who contribute to both public goods. Of course a special case would be Kemp's

\footnotetext{
${ }^{7}$ These are particularly treacherous when inequality constraints are lurking. In fairness, we must emphasize that both Warr and Kemp qualify their statements appropriately.

${ }^{8}$ Warr's theorem, although based on first-order conditions, does not require that all consumers contribute positive amounts, only that the redistribution be solely among contributors. This escape is not available for Kemp's theorem in its current form since the normal case would seem to be where some people contribute to one of the goods and not to the other.
} 
case of a redistribution among all consumers where all consumers contribute to every public good. Where the quantity of one public good affects the demand for the other public good it is easy to see why some such restriction is needed. For example, if the sets of contributors to $G$ and $H$ are neither disjoint nor identical, then some redistributions of income among contributors to $G$ may increase total income of contributors to $H$ resulting in an increased supply of $H$ and consequent feedback on contributions to $G$.

Theorem 7 can be extended to the case of more than two public goods, where the class of income redistributions considered is such that for every subset of the set of all public goods, aggregate income is constant for the set of consumers who contribute positive amounts to these goods and nothing to any other public goods. In general, if there are many public goods, this is a very restrictive condition. However, in many applications, there is special structure such as the sets of contributors to certain public goods being identical or disjoint which makes this restriction quite natural.

\section{Further applications}

Extending our results to the case of several public goods allows a number of interesting applications. One application, suggested by the work of Arrow (1981) and Becker (1981), concerns the case of voluntary income redistribution. If some people are altruistically concerned about the consumptions of others, we can model the consumption of each individual in whom others are interested as a pure public good. (There is no loss of generality in doing so even if some consumers are totally uninterested in this individual's consumption, since the theory of public goods remains intact although some of the public goods may enter some utility functions in a trivial way.) If the population can be partitioned into 'families' in such a way that all contributors to any individual are in his own family, then Theorem 7 could be applied to redistributions of income within families.

Another example is the case of two rival political parties. Suppose that the probability that either party achieves office depends positively on the total amount of campaign contributions that it receives and negatively on the total amount of contributions that its rival receives. Contributions to both parties can be treated as 'pure public goods', where adherents of either party regard contributions to their own party as desirable and contributions to the other party as undesirable. In this model, one's willingness to contribute to his own party depends both on the sum of contributions to his own party and on the sum of contributions to the other party. Theorem 7 informs us that a small redistribution of income among contributors to either party will not change total contributions either to that party or to its rival.

Yet a third application is to the macroeconomic 'neutrality' literature initiated by Barro (1974). Bernheim and Bagwell (1984) provide a very 
general treatment of redistributive neutrality in this context, both across and within generations. However, they, along with the others who have written in this area, examine only the interior case - where every agent is contributing a positive amount to his descendants. The results presented earlier in this paper suggest that the analysis of the 'boundary case', where some agents are not contributing, may well be tractable. Furthermore, we would conjecture that when such boundary cases occur there will be significant nonneutralities of the sort we have found for our problem. The decision of whether or not to contribute - to a public good, or to one's descendants - is an endogenous one, and the adjustment on the 'extensive margin' is at least as important as the adjustment on the 'intensive margin'.

\section{References}

Abrams, B. and M. Schmitz, 1978, The 'crowding-out' effect of government transfers on private charitable contributions, Public Choice 33, 29-41.

Andreoni, J., 1985, A theory of private donations to public goods: The egoistic contributor model, University of Michigan Working Paper.

Arrow, K., 1981, Optimal and voluntary income redistribution, in: S. Rosefielde, ed., Economic welfare and the economics of soviet socialism: Essays in honor of Abram Bergson, Cambridge University Press, 1981. Reprinted in K. Arrow, Collected papers of Kenneth J. Arrow, v. 1, Harvard University Press, 1983.

Barro, R., 1974, Are government bonds net wealth?, Journal of Political Economy 82, 10951117.

Becker, G., 1981, A treatise on the family, Harvard University Press, Cambridge.

Bergstrom, T. and H. Varian, 1985, When are Nash equilibria independent of the distribution of agents' characteristics?, Review of Fconomic Studics 52, 715-718.

Bernheim, B. and K. Bagwell, 1984, Is everything neutral? The implications of intergenerational altruism in an overlapping generations model with marriage, Stanford University Working Paper.

Borcherding, T., 1977, The sources of growth of public expenditures in the United States, 19021970, in: Borcherding, ed., Budgets and bureaucrats: The sources of government growth, Duke University Press, Durham, N.C.

Brennan, G. and J. Pincus, 1983, Government expenditure growth and resource allocation: The nebulous connection, Oxford Economic Papers 35, 351-365.

Chamberlin, J., 1974, Provision of collective goods as a function of group size, American Political Science Review 68, 707-716.

Chamberlin, J., 1976, A diagrammatic exposition of the logic of collective action, Public Choice $26,59-74$.

Cornes, R. and T. Sandler, 1984, Easy riders, joint production, and public goods, Economic Journal 94, 580-598.

Cornes, R., and T. Sandler, 1985, The simple analytics of pure public good provision, Economica 52, 103-116.

Isaac, M., K. McCue and C. Plott, 1982, Nash equilibrium in public goods provision: Free riding in experimentally controlled markets, California Institute of Technology Social Science Working Paper No. 428.

Kemp, M., 1984a, A note on the theory of international transfers, Economic Letters 14, 2-3, $259-262$.

Kemp, M., 1984b, On the applicability and implementability of the finite compensation principle, Kobe Economics and Business Review.

Kim, O. and M. Walker, 1984, The free rider problem: Experimental evidence, Public Choice 43, $3-24$. 
Laffont, J., 1982, Cours de theorie microeconomique 1, Fondements de l'economie publique, Economica, Paris.

Marwell, G. and R. Ames, 1981, Economists free ride, does anyone else?, Journal of Public Economics 15, 295-310.

McGuire, M., 1974, Group homogeneity and aggregate provision of a pure public good under Cournot behavior, Public Choice 18, 107-126.

Olson, M., 1965, The logic of collective action, Harvard University Press, Cambridge.

Roberts, R., 1984, A positive model of private charity and wealth transfers, Journal of Political Economy 92, 136-148.

Smith, V., 1980, Experiments with a decentralized mechanism for public good decisions, American Economic Review 70, 584-599.

Steinberg, B., 1984, Voluntary donations and public expenditures, V.P.I. and S.U. Economics Department Working Paper, Blacksburg, Virginia.

Sugden, R., 1982, On the economics of philanthropy, Economic Journal , 341-350.

Warr, P., 1982, Pareto optimal redistribution and private charity, Journal of Public Economics $19,131-138$

Warr, P., 1983, The private provision of a public good is independent of the distribution of income, Economic Letters 13, 207-211.

Young, D., 1982, Voluntary purchase of public goods, Public Choice 38, 73-86. 